\title{
Uso de Cratylia argentea en la zona tropical de Colombia
}

\author{
Use of Cratylia argentea in the tropical zone of Colombia \\ Plazas Borrero Camilo Hernando ${ }^{1}$ \\ ${ }^{1}$ MVZ. Esp. MSc. Docente Universidad de los Llanos \\ cplazasb@unillanos.edu.co
}

Recibido 08 de Septiembre 2014, Aceptado 10 de Abril 2015

\section{RESUMEN}

La alimentación de vacas lecheras en sistemas doble propósito en los Llanos orientales de Colombia, se basa principalmente en pasturas de Brachiaria, las cuales se encuentran en diferentes estados de degradación. Una limitación tanto en época de lluvia como sequia es el bajo contenido de proteína en el forraje lo cual determina la baja producción de leche y necesidad de suplementar durante la época seca con concentrado si se quiere mantener la producción, en consecuencia, la eficiencia biológica y económica de estos sistemas es baja, lo cual afecta negativamente los ingresos y competitividad de los productores de carne y leche. Para contribuir a mejorar los recursos alimenticios en pequeñas fincas lecheras, se ha llevado a cabo varios proyectos para validar y promover el uso de Cratylia argentea (veranera). En un primer proyecto se seleccionaron 14 fincas cuya actividad principal era la producción de leche, con la participación activa de los productores se establecieron 11.8 ha, de las cuales 6.5 fueron destinadas para corte y acarreo, 3 para ramoneo y 2.26 para producción de semillas. En forma paralela, se realizaron trabajos de uso de Cratylia en el "Centro de Investigaciones La libertad"; Colonia penal de Acacias, además se establecieron 2 ha en la finca Santana situada en la altillanura plana, 20 ha en ocho colegios agropecuarios de la región y varios lotes de producción de semillas con destino al fomento de la veranera entre productores. Además, se realizó el ejercicio de la creación de la Red Nacional de Productores de Cratylia, que tuvo sus ensayos en diferentes zonas agroecológicas del país. Tanto en las fincas como en los colegios, los productores aportaron la mano de obra necesaria para el 
establecimiento y el mantenimiento del cultivo. En la época lluviosa la producción de veranera en las fincas fue, en promedio, de 3.34 ton/ha de materia seca (MS) con un $66 \%$ de hoja y una altura de planta de $137 \mathrm{~cm}$. Durante el periodo seco la producción fue de 0.59 ton/ha de MS con $52 \%$ de hoja y una altura de $112 \mathrm{~cm}$. El contenido de proteína cruda $(\mathrm{PC})$ de veranera durante la época seca fue, en promedio $21.67 \%$, mientras que en la gramínea acompañante fue de $6.52 \%$. Los rendimientos de semillas limpia en lotes establecidos en siete fincas fueron menores a lo inicialmente esperado; en la primera época seca se produjeron únicamente de $33 \mathrm{~kg} / \mathrm{ha}$, equivalente a $47 \mathrm{~g} /$ planta, siendo significativamente inferiores a los rendimientos obtenidos en Costa Rica con esta misma leguminosa. Los resultados han mostrado que es una excelente alternativa para aumentar la productividad animal en los Llanos Orientales y permite incrementar la producción de carne por hectárea y por año, en comparación con el sistema tradicional de solo pastura.

Palabras clave: Arbustiva forrajera, suplementación, leguminosa, alimentación.

\begin{abstract}
Feeding dairy cows in dual purpose systems in the eastern plains of Colombia, it is mainly based on Brachiaria pastures, which are in different stages of degradation. A limitation in both rainy and drought is the low protein content in the forage which determines the low production of milk and need to supplement during the dry season to concentrate if we want to maintain production therefore biological efficiency and economic of these systems is low, which negatively affects revenues and competitiveness of producers of meat and milk. To help improve food resources in small dairy farms, it has carried out several projects to validate and promote the use of Cratylia (veranera). In a first project 14 farms were selected whose main activity was the production of milk, with the active participation of producers 11.8 ha were established, of which 6.5 were designed to cut and carry, 3 for browsing and 2.26 for seed production. In parallel, we work Cratylia use the "Research Center La Libertad" were performed; Acacias penal colony also was established 2 ha in the Santana farm in the flat altillanura, 20 has in eight
\end{abstract}


agricultural schools in the region and several batches of seed production destined to the promotion of bougainvillea between producers. Hence the exercise of the creation of the National Network Cratylia Producers, which had its trials in different agro-ecological zones of the country was conducted. Both on farms and in schools, the producers supplied the labor required for the establishment and maintenance of crops. In the rainy season Cratylia production on farms it was on average 3.34 ton/ha of dry matter (DM) with $66 \%$ leaf and plant height of $137 \mathrm{~cm}$. During the dry season production was 0.59 ton/ha of DM with $52 \%$ leaf and a height of $112 \mathrm{~cm}$. The crude protein content (PC) of Cratylia during the dry season averaged $21.67 \%$, while the companion grass was $6.52 \%$. Yields of clean seeds in batches established in seven farms were lower than initially expected; in the first dry season there were only $33 \mathrm{~kg} / \mathrm{ha}$, equivalent to $47 \mathrm{~g} / \mathrm{plant}$, being significantly lower than the yields obtained in Costa Rica with the same legume. The results have shown that it is an excellent alternative for increasing productivity in the Eastern Plains and allows to increase meat production per hectare per year, compared with the traditional system of pasture alone.

Keywords: Shrubby forage, supplementation, legume, feeding.

\section{RESUMO}

Alimentação de vacas leiteiras em sistemas de duplo propósito nas planícies orientais da Colômbia, é baseado principalmente em pastagens de Brachiaria, que estão em diferentes estágios de degradação. Uma limitação em ambos chuvosa e seca é o baixo teor de proteína na forragem que determina a baixa produção de leite e precisam complementar durante a estação seca para se concentrar se queremos manter a produção biológica e, portanto, a eficiência económico destes sistemas é baixa, o que afeta negativamente a receita e competitividade dos produtores de carne e leite. Para ajudar a melhorar os recursos alimentares em pequenas explorações leiteiras, tem realizado vários projectos para validar e promover o uso de Cratylia (buganvílias). Em um primeiro projeto de 14 fazendas foram selecionados cuja actividade principal era a produção de leite, com a participação ativa dos produtores de 11.8 ha foram estabelecidos, dos quais 6.5 
foram projetados para corte e transporte, 3 para a navegação e 2.26 para produção de sementes. Em paralelo, trabalhamos Cratylia usar o "Centro de Pesquisa Liberdade" foi executada; Acacias colônia penal também estabeleceu 2 ha na fazenda Santana no apartamento altillanura, 20 ha em oito escolas agrícolas da região e vários lotes de produção de sementes destinadas à promoção da buganvília entre os produtores. Por isso, o exercício da criação da rede Cratylia Produtores nacionais, que tiveram seus ensaios em diferentes zonas agroecológicas do país foi conduzido. Tanto nas explorações agrícolas e nas escolas, os produtores forneceram a mão de obra necessária para o estabelecimento e manutenção de culturas. Na estação chuvosa Veranera produção nas fazendas era, em média, 3.34 ton/ha de matéria seca (MS), com $66 \%$ de folhas e altura de planta de $137 \mathrm{~cm}$. Durante a estação seca a produção foi de 0.59 ton/ha de MS com $52 \%$ de folhas e uma altura de $112 \mathrm{~cm}$. O teor de proteína bruta (PC) de Veranera durante a estação seca em média $2.67 \%$, enquanto a grama companheiro era $6.52 \%$. Os rendimentos de sementes limpas em lotes estabelecidos em sete fazendas foram menores do que o inicialmente esperado; na primeira estação seca, havia apenas $33 \mathrm{~kg} / \mathrm{ha}$, o equivalente a $47 \mathrm{~g} /$ planta, sendo significativamente inferior aos rendimentos obtidos em Costa Rica com o mesmo legume. Os resultados têm mostrado que é uma excelente alternativa para o aumento da produtividade nas planícies orientais e permite aumentar a produção de carne por hectare por ano, em comparação com o sistema tradicional de pasto sozinho.

Palavras-chave: Forragem arbustiva, suplementação, legume, alimentação.

\section{INTRODUCCIÓN}

El sistema de producción con bovinos doble propósito tiene por objetivo producir tanto leche como carne (Cortés et al., 2012). En Colombia el 93\% del inventario ganadero se encuentra bajo este sistema (MADR y CCl, 2009), el cual aporta el $50 \%$ de la producción láctea, proveniente de sistemas doble propósito localizadas a menos de 1000 m.s.n.m (CCl y MADR, 2010). En los Llanos Orientales la productividad es baja: entre 2 y 4 L/vaca por día, natalidad de 40 a $50 \%$ y un peso 
vivo animal desteto entre 100 a $120 \mathrm{~kg}$ (FEDEGAN, 2006). Esta baja productividad se debe a la deficiente calidad nutritiva de las pasturas, principalmente durante la época seca. Una alternativa comúnmente utilizada por los productores para hacer frente a esta situación es el suministro de concentrados comerciales de alto costo (Valencia et al., 2010).

En los Llanos Orientales de Colombia predominan las especies de Brachiaria en sistemas de producción doble propósito las cuales, en alta proporción, se encuentran en diferentes estados de degradación y consecuentemente su calidad nutritiva y consumo por los animales son muy bajos. Una alternativa para suplementar proteína a los animales es la introducción de leguminosas forrajeras (Valencia et., 2010). Los estudios previos mostraron que algunas de ellas, como Leucaena leucocephala y Gliricidia sepium, no se adaptan a los suelos ácidos de baja fertilidad comunes en esta zona, no obstante, el Programa de Forrajes Tropicales del CIAT después de evaluar 22 especies arbustivas y arbóreas identificó a Cratylia argentea (veranera) como una de las especies mejor adaptadas en fincas con sistemas doble propósito bajo las condiciones del Piedemonte (Lascano et al., 2002).

El objetivo del trabajo fue fomentar el uso de veranera entre productores con el fin de contribuir a aumentar sus ingresos y a una mayor competitividad del sector lechero de los Llanos Orientales de Colombia. También se quiso determinar las ventajas biológicas y económicas del uso esta leguminosa como suplemento para vacas lecheras y establecer metodologías de transferencia de esta tecnología entre productores de la región.

Los trabajos fueron parte de un proyecto financiado por el Ministerio de Agricultura de Colombia y el Centro Internacional de Agricultura Tropical y fueron realizados en las veredas La Llanerita, El Hachón y Santa Helena, en la vía VillavicencioPuerto López, departamento del Meta, Colombia. Los sitios de los ensayos se encuentran a 305 m.s.n.m., con una temperatura de $27^{\circ} \mathrm{C}$ y $3650 \mathrm{~mm}$ de precipitación anual, una humedad relativa del 75\% y un brillo solar de 1467 horas luz por año. Los suelos están constituidos principalmente por terrazas altas y 
bajas, donde las primeras se caracterizan por ser arcillosos ácidos con alto contenido de aluminio y poca fertilidad natura, y han sido intervenidos por el hombre para el establecimiento de pasturas y cultivos comerciales (Plazas y Lascano, 2005).

\section{Uso del suelo y sistemas de producción}

En las fincas donde se realizaron los trabajos predomina la ganadería doble propósito en 7300 ha de Brachiaria decumbens y humidicola. Los cultivos permanentes comprenden 213 y perennes 182 ha, los bosques de galería se encuentran en 180 ha. El número de fincas en las veredas del estudio es de 106 y el $57 \%$ de ellas tienen entre 1 y 20 ha. La ganadería en el área de estudio se basa en la producción de leche y carne, incluyendo la cría, el levante y la ceba de bovinos. Las explotaciones son extensivas en sabanas bien drenadas de Trachypogon sp., Axonopus purpusi, Paspalum contractum y Andropogon gayanus, especies nativas de baja producción y calidad que son sometidas a quemas frecuentes. En las explotaciones lecheras se hace un ordeño diario en las horas de la mañana, la leche así como los terneros machos, los novillos cebados y las vacas de descarte son vendidos en el mercado local.

\section{METODOLOGÍA}

En una encuesta previa se identificaron las necesidades y las posibilidades de adopción de nuevas tecnologías basadas en el uso de leguminosas forrajeras semiarbustivas. No obstante la baja aceptación inicial del cv. Veranera, fue posible seleccionar 14 productores con hatos destinados a la producción en sistema doble propósito que estaban dispuestos a ensayarlo en sus fincas. En total fueron establecidas 11.8 ha, 6.5 para corte y acarreo, 3 para ramoneo y 2.26 para producción de semillas. En forma paralela con los trabajos en las fincas, en ocho colegios agropecuarios de la región, se establecieron lotes de producción de semillas con destino al fomento de la veranera entre los ganaderos. En fincas y colegios, los productores aportaron la mano de obra necesaria para el establecimiento y el mantenimiento del cultivo. En los lotes destinados a corte y 
acarreo la siembra se hizo a 'chuzo' a una distancia de $1 \mathrm{~m}$ entre plantas y entre surcos. Cuando los lotes se destinaron a la producción de semillas, la siembra se hizo a $1.5 \mathrm{~m}$ y una vez germinaron se procedió a la resiembra en aquellos sitios donde la población de plántulas fue baja. Para el control de malezas en posemergencia se utilizó glifosato y fertilizando en cada planta $15 \mathrm{~g}$ de urea y una mezcla de superfosfato triple $(10 \mathrm{~g}), \mathrm{KCl}(10 \mathrm{~g})$, cal dolomítica (14 g ), sulfato de magnesio (8 $\mathrm{g}$ ), flor de azufre $(1 \mathrm{~g})$, de acuerdo con los resultados del análisis de suelo. Una vez que los lotes se sembraron, los productores en cada sitio fueron responsables de su mantenimiento y utilización. Como parte del programa de asesoramiento, los técnicos de las instituciones involucradas en el proyecto realizaron visitas periódicas para hacer observaciones y evaluar conjuntamente con los ganaderos el comportamiento del cultivo de la veranera.

\section{Establecimiento}

El establecimiento en franjas de $C$. argentea fue difícil debido a la alta competencia con la gramínea y malezas, por tanto, en los sistemas de ramoneo es recomendable sembrar primero la leguminosa y posteriormente la gramínea. El comportamiento de la veranera en los lotes para corte y acarreo y en los semilleros fue exitoso (Figura 1), aunque en algunos de ellos se presentaron problemas de malezas, encharcamientos y plagas, que fueron fácilmente controlados. La siembra por trasplante representó un alto costo por el mayor uso de mano de obra, además de un daño radicular a las plántulas que retardó su desarrollo.
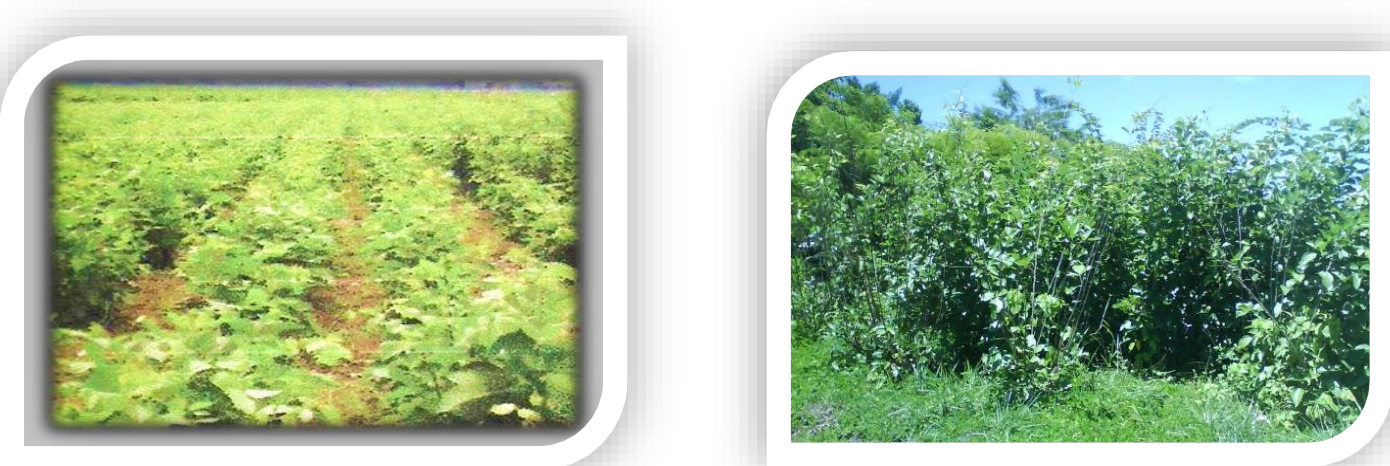

Figura 1. Establecimiento Cratylia argentea en época seca y de lluvia 


\section{PRODUCCIÓN DE FORRAJE Y ALTURA DE PLANTA}

El primer corte se realizó 6 meses después de la siembra a una altura entre 20 y $30 \mathrm{~cm}$ sobre el nivel del suelo, se obtuvo en promedio de producción 2.01 ton/ha de MS con una relación hoja: tallo de 2:1 y una altura de planta de $121 \mathrm{~cm}$. El segundo corte fue realizado 70 días más tarde, alcanzándose una producción promedio por finca, de 1.96 ton/ha de MS y una altura de $122 \mathrm{~cm}$.En la época Iluviosa la MS obtenida en las fincas fue 3.34 ton/ha con un $66 \%$ de hoja y una altura de $137 \mathrm{~cm}$, siendo la producción en el periodo seco de 0.59 ton/ha de MS con $52 \%$ de hoja y una altura de $112 \mathrm{~cm}$. A pesar de la alta diferencia entre los resultados de las dos épocas, fue posible observar un buen rebrote y persistencia de las plantas en el periodo seca.

\section{CALIDAD NUTRITIVA}

El contenido de proteína cruda (PC) de veranera durante la época seca fue, en promedio $21.67 \%$, mientras que la gramínea presentó tan solo $6.52 \%$. El mayor contenido de PC de esta leguminosa es una de las características sobresalientes que justifica su inclusión como una alternativa de suplementación para reemplazar el uso de concentrados durante la época seca en sistemas doble propósito de la región. Es importante mencionar que el contenido de PC puede llegar hasta 10\% en ensilado de maíz con veranera Vs 7\% con solo maíz.

\section{SISTEMAS DE USO}

Inicialmente los productores utilizaron corte y acarreo para proporcionar el forraje picado en canoas al momento del ordeño. Posteriormente, y debido a los altos costos de este sistema, los mismos productores cosecharon el forraje y lo dejaron sobre las pasturas a libre disposición de los animales después del ordeño. Finalmente, decidieron que la mejor forma de suministrar la veranera era mediante el pastoreo directo en forma de ramoneo (Figura 2), con este último sistema han logrado reducir los costos de mano de obra y hacer un uso más racional de la leguminosa. No obstante, cuando las plantas no se cortan uniformemente después del ramoneo presentan una baja proporción de material aprovechable como hojas 
(29\%) en el siguiente período de uso vs. plantas cosechadas a $20 \mathrm{~cm}$ sobre el suelo, que presentan $54 \%$ de hojas.

\section{PRODUCCIÓN DE LECHE}

Aunque el aumento en la producción de leche durante la época seca debido al suministro de veranera no fue tan significativo, los productores coincidieron en que su suministro les permitió mantener el nivel de producción y la venta de leche en el mercado local, así como la reducción en la compra de concentrados comerciales, lo cual no era posible antes de la introducción de este nuevo recurso forrajero. Además de las ventajas en el sostenimiento de la producción de leche durante la época seca, los productores reconocieron el mejor desarrollo y comportamiento reproductivo de las vacas suplementadas con esta leguminosa.

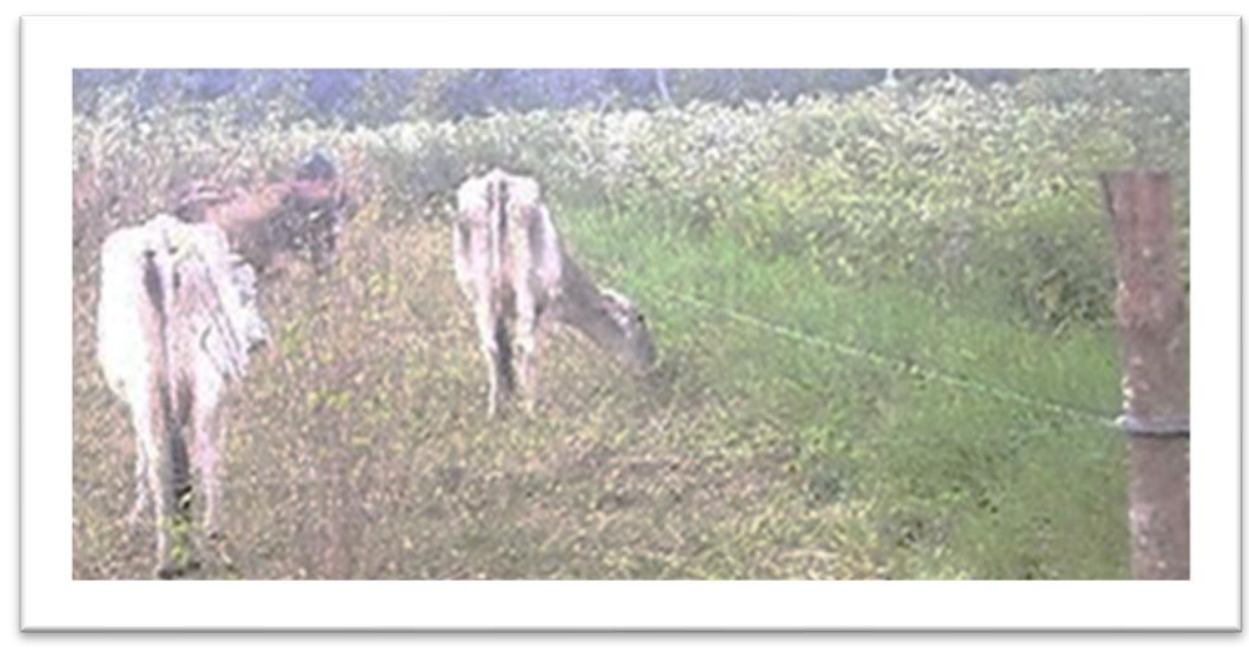

Figura 2. Ramoneo directo de Cratilia argéntea en época seca

\section{PRODUCCIÓN DE SEMILLAS}

Los rendimientos de semillas limpia en lotes establecidos en siete fincas fueron más bajos que lo inicialmente esperado; en la primera época seca se produjeron únicamente $33 \mathrm{~kg} / \mathrm{ha}$, equivalente a $47 \mathrm{~g} /$ planta, siendo significativamente inferior a los rendimientos obtenidos en Costa Rica con esta misma leguminosa (Argel et al., 2002). 
En el período Junio-Julio del segundo año del cultivo se hizo un corte de uniformización y se aplicaron $70 \mathrm{~g} /$ planta de un fertilizante completo, el cual floreció en Diciembre y la recolección de las semillas maduras se hizo en Febrero del año siguiente. Aunque inicialmente la calidad de las semillas era buena $(90 \%$ de germinación) posteriormente, en Abril, al comienzo de la época de lluvias, este se redujo drásticamente alcanzando un valor de $40 \%$. Los resultados de estos trabajos confirman nuevamente las desventajas de los Llanos Orientales de Colombia como región adecuada para la producción comercial de semillas de veranera. Entre los factores responsables de los bajos rendimientos se pueden citar la baja polinización natural, la ocurrencia ocasional de lluvias durante la época seca que favorece una mayor humedad relativa, vientos fuertes que provocan defoliación y caída de flores y el alto costo de la mano de obra para realizar estas labores.

\section{LECCIONES APRENDIDAS}

La participación activa de los productores permitió identificar formas de cultivo y de uso de veranera, diferentes a aquellos inicialmente propuestos en estos trabajos. El sistema de corte y acarreo resultó ser costoso debido a la alta demanda de mano de obra, por tanto, los productores prefieren utilizar el suministro del forraje directamente en el campo, ramoneo y ensilaje. Fue interesante observar que los productores establecen el veranera intercalado con cultivos de maíz u hortalizas, lo que les permite reducir costos en esta fase del cultivo. Inicialmente se propuso que la veranera fuera una fuente para la alimentación en épocas secas de vacas en producción, no obstante, los productores encontraron ventajas en el uso de esta leguminosa para: (1) la alimentación de vacas en épocas lluviosas cuando el exceso de humedad no permite el pastoreo de los animales; (2) reemplazar parte de los concentrados comerciales que suministran a las vacas lactantes; (3) mantener la producción de leche del hato durante las épocas secas y de exceso de humedad; (4) el mantenimiento de las condiciones corporales y reproductivas de los animales. Experiencias de campo en la Colonia Agrícola donde el primer corte se realizó entre los 6 y 8 meses a una altura de 1 metro sobre el suelo, los 
aportes en tallos y hojas estuvieron alrededor de 0.7 a $0.9 \mathrm{~kg}$ forraje verde/planta, de esta manera el forraje ofertado en el primer corte esta alrededor de 0.7 a $0.9 \mathrm{~kg}$ forraje verde/planta. Los cortes posteriores se realizan entre 50 y 90 días a una altura de $50 \mathrm{~cm}$. En la Colonia agrícola en plantas con rebrotes de 55 días se obtuvieron de 1 a $1.2 \mathrm{~kg}$ forraje verde/planta y en rebrotes de 90 días el rendimiento fue de $2 \mathrm{~kg}$. En época en la que escasea el alimento se ha ensilado Cratylia con una participación del 30\%, el 70\% corresponde a el uso de gramíneas de corte y el $2 \%$ con melaza; esto con el fin de proporcionar carbohidratos solubles que le permitan al proceso de fermentación ser más eficiente. Se asume que una planta en términos generales bien desarrollada produce entre 0.6 a $1 \mathrm{~kg}$ de material aprovechable cada 60 o 90 días; el consumo diario por vaca en ordeño varía entre 6 y $10 \mathrm{~kg}$ de forraje fresco, más 10 a $15 \mathrm{~kg}$ de pastos de corte; es decir, que la mezcla de la ración puede contener $60 \%$ de pastos de corte y $40 \%$ de Cratylia. De lo anterior se deduce que es necesario cosechar diariamente entre 6 y 10 plantas del arbusto para alimentar una vaca, por lo tanto esta leguminosa también se puede utilizar para fabricar ensilajes con gramíneas o una fuente de energía como caña de azúcar.

Investigadores en nutrición animal de Corpoica, han realizado trabajos donde se ha utilizado hasta un $50 \%$ Cratylia y $50 \%$ caña de azúcar, logrando un buen ensilaje. Lo más recomendable para no correr riesgos es utilizar $30 \%$ Cratylia y $70 \%$ caña de azúcar o cualquier otra gramínea. En el Centro de Investigaciones La Libertad, se encontró que la suplementación de $25 \%$ de Cratylia más $75 \%$ de caña a novillos jóvenes Cebú puro y cruzado con Sanmartinero en pasturas de $B$. decumbens resulto en una mayor producción de carne por ha/año en comparación con animales únicamente en pastoreo. Animales sin suplementación, la carga fue 2.5 animales/ha, ganancia animal/día de $545 \mathrm{~g}$ en los Cebú y $696 \mathrm{~g}$ en los Cebú $\mathrm{x}$ Sanmartinero, logrando un total de $\mathrm{kg} / \mathrm{ha} / \mathrm{año}$ de $498 \mathrm{~kg}$ y $635 \mathrm{~kg}$ de carne respectivamente. En los lotes de animales suplementados, se logró subir la carga a 5 animales/ha, con ganancia animal/día de $679 \mathrm{~g}$ en los Cebú y $624 \mathrm{~g}$ en los cruzados, arrojando un total de kg/ha/año de $1239 \mathrm{~kg}$ y $1139 \mathrm{~kg}$ de carne respectivamente (Lascano et al, 2002). 
En la Finca Santana se elaboró un ensilaje con 30\% Cratylia y $70 \%$ Pasto maralfalfa (Pennisetum ssp). Las 20 ha de esta leguminosa sembradas en esta finca fueron utilizadas para ensilajes con caña y pasto maralfalfa teniendo como finalidad suministrar este alimento a animales en confinamiento. La adopción de $C$. argentea en los Llanos Orientales de Colombia es un proceso continuo que está siendo promovido por los técnicos en extensión de instituciones nacionales capacitadas para esta labor y por productores entusiastas que han reconocido los beneficios de la leguminosa en sus fincas. Sin embargo, la adopción de este cultivar en varias regiones de Colombia es un proceso lento debido principalmente a la falta de semilla comercial.

\section{CONCLUSIONES}

Aunque el aumento en la producción de leche durante la época seca debido al suministro de veranera no fue significativo, los ganaderos coincidieron en que si les permitió mantener el nivel de producción y la venta de leche en el mercado local, así como la reducción en la compra de concentrados comerciales, lo cual no era posible antes de la introducción de este nuevo recurso forrajero.

Además de las ventajas en el sostenimiento de la producción de leche durante la época seca, los productores reconocieron la buena condición corporal y su efecto positivo en la reproducción (presentación de calores más rápidos en época seca, disminución de vacas repetidoras de calor). La posibilidad de tener un forraje de alta calidad durante la estación lluviosa, cuando se dificulta pastorear debido a la alta humedad en los suelos, en forma de: corte y acarreo, ensilaje, henolaje, pastoreo directo con el uso de cercas eléctricas acompañadas de gramíneas, reemplazo de concentrados utilizados en la estación seca, con las implicaciones de disminuir costos, y posibilidad de ordeñar las consiguiendo un precio superior para la leche vendida.

\section{REFERENCIAS BIBLIOGRÁFICAS}

1. Argel P, Lobo M, Hidalgo C, González J, Jiménez C. Cratylia argentea cultivar Veraniega: Una leguminosa arbustiva para la ganadería del trópico de América Latina. Boletín de Divulgación. Ministerio de Agricultura y Ganadería (MAG), 
Escuela Centroamericana de Ganadería (ECAG), Universidad de Costa Rica y Centro Internacional de Agricultura Tropical (CIAT). San José, Costa Rica. 20 p. 2001.

2. Argel P, Giraldo G, Peters M, Lascano CE. Producción artesanal de semillas de cratylia (Cratylia argéntea) accesiones CIAT 18516 y 186668. Centro Internacional de Agricultura Tropical (CIAT); Bundesministerium für Wirtschaftliche Zusammenarbeit und Entwicklung (BMZ); Deutsche Gesellschaft für Technische Zusammenarbeit (GTZ), 12 p. 2002.

3. $\mathrm{CCl}$ (Corporación Colombia Internacional), y MADR (Ministerio de Agricultura y Desarrollo Rural de Colombia). Caracterización de la producción de leche en Colombia. 1aㅡ ed. Bogotá, Colombia. 2010.

4. Cortés JA, Cortés A, Cotes JM. Características estructurales del sistema de producción con bovinos doble propósito en el trópico húmedo colombiano. Rev Col Cien Pec., 25 (2): 229-239. 2012.

5. FEDEGAN (Federación Colombiana de Ganaderos). Plan estratégico de la ganadería colombiana 2019. Por una ganadería moderna y solidaria. Bogotá, Colombia, 294 p. 2006.

6. Holmann F, Lascano C. Sistemas de alimentación con leguminosas para intensificar fincas lecheras. Un proyecto ejecutado por el Consorcio Tropileche. Centro Internacional de Agricultura Tropical (CIAT). Cali, Colombia. 2001.

7. Lascano C. Calidad nutritiva y utilización de Cratylia argentea. En: Pizarro E y Coradin L (eds.). Potencial del género Cratylia como leguminosa forrajera. Memorias del taller de trabajo sobre Cratylia. EMBRAPA, CENARGEN, CPAC, y CIAT. Brasilia, Brasil. 1995.

8. Lascano C, Rincón A, Plazas C, Avila P, Bueno G, Argel PJ. Cultivar Veranera (Cratylia argéntea (Desvaux) $O$. Kuntze), Leguminosa arbustiva de usos múltiples para zonas con períodos prolongados de sequía en Colombia: Villavicencio, Colombia: Corporación Colombiana de Investigación agropecuaria, Centro Internacional de Agricultura Tropical, Cali, Colombia. 28 p. 2002.

9. MADR (Ministerio de Agricultura y Desarrollo Rural de Colombia) y $\mathrm{CCl}$ (Corporación Colombia Internacional). Oferta agropecuaria: Encuesta Nacional Agropecuaria. Bogotá, Colombia. 2009.

10. Plazas $\mathrm{CH}$, Lascano $\mathrm{CE}$. Utilidad de Cratylia argentea en ganaderías de doble propósito del piedemonte de los llanos orientales de Colombia. Pasturas Trop 27 (2): 65-72. 2005.

11. Valencia L, Restrepo J, Cerón D, Herrera W. Determinación de la digestibilidad in vivo en ovinos utilizando dietas a base de forrajes tropicales. Revista de Investigación Agraria y Ambiental, 1 (1): 25-29. 2010. 